\title{
Construcción de una base de datos electrocardiográfica para caninos residentes en Bogotá, con apoyo ecocardiográfico
}

\author{
Mauricio Ortega ${ }^{1}$, Leonardo Gómez ${ }^{1}$, Alexander Cerquera ${ }^{2,3}$ y Rafael Gutiérrez ${ }^{3}$ \\ ${ }^{1}$ Facultad de Medicina Veterinaria, ${ }^{2}$ Facultad de Ingeniería Electrónica y Biomédica, ${ }^{3}$ Grupo de Sistemas Complejos Universidad \\ Antonio Nariño - Bogotá. \\ Correspondencia: mauricio.ortega@uan.edu.co
}

Recibido:12-04-2006 Aceptado:04-07-2006

\begin{abstract}
Resumen
El siguiente trabajo presenta la base de datos VETUAN_I, de medidas obtenidas en datos de reseña, electrocardiogramas y ecocardiogramas correspondientes a pacientes caninos sin deficiencias cardiacas evaluados en la Clínica Veterinaria "Veteriland" de la ciudad de Bogotá, mediante la utilización de equipos de diagnóstico de la Universidad Antonio Nariño. Esta base de datos está constituida por los parámetros o medidas cardiacas más utilizadas y por otros adicionales, lo cual permite comparar, revaluar y ajustar los valores estándares establecidos desde los años 60, comprender mejor su utilidad de diagnóstico, realizar análisis comparativos entre razas y otros factores, así como establecer diferentes correlaciones que mejoren la capacidad de diagnóstico.
\end{abstract}

Palabras clave: electrocardiograma, ecocardiograma, diagnóstico, parámetros cardiacos, caninos.

\begin{abstract}
Construction of an electrocardiographic data base for canine residents in Bogota, with ecocardiographic support: The following work presents the data base VETUAN_I, of measures obtained in data of review, electrocardiograms and ecocardiograms corresponding to canine patients without evaluated cardiac deficiencies in the Veterinary Clinic «Veteriland» of the city of Bogota, by means of the use of equipment of diagnosis of the Antonio Nariño University. This data base is constituted by the cardiac parameters or cardiac measures more used and by other additional ones, which allows to compare, to revalue and to fit the established standard values from 60's, to understand better its utility of diagnosis, to make comparative analyses between races and other factors, as well as to establish different correlation that improve the diagnosis capacity.
\end{abstract}

Key words: electrocardiogram, ecocardiogram, diagnosis, cardiac parameters, canine

\section{Introducción}

La electrocardiografía es una técnica que sirve para evaluar la actividad eléctrica del corazón, por lo que es $99 \%$ efectiva para detectar arritmias cardiacas pero sólo tiene un 60 a $70 \%$ de efectividad para detectar cardiomegalias, por lo cual, es necesario recurrir a otras pruebas diagnósticas complementarias como la radiología, la ecocardiografía, la angiografía y el doppler, para llegar al diagnóstico final (1). 
La utilización de las pruebas complementarias cubre ese 30 a $40 \%$ de las causas de cardiopatías en caninos que no se pueden diagnosticar con sólo el electrocardiograma (ECG). Adicionalmente, la complementariedad entre diferentes exámenes tiene una capacidad de diagnóstico y comprensión de problemas cardiacos que no tiene ningún examen por separado. En el ECG se realizan mediciones de amplitud y duración de las diferentes ondas y segmentos de los registros, así como del eje eléctrico promedio, lo que al interpretarlo en conjunto sirve para inferir un probable agrandamiento de las cámaras del corazón. Se debe recurrir a la radiología para corroborar o descartar estos probables agrandamientos por medio de técnicas directas e indirectas (2). La utilización en conjunto de la electrocardiografía con la ecocardiografía, permiten obtener mayor precisión en el diagnóstico, por supuesto sin dejar de lado, el examen físico, que sigue siendo sin duda, el arma diagnóstica más importante.

En la revisión del estado de arte se percibe que la tabla de valores electrocardiográficos de caninos utilizada en la práctica clínica, no se ha modificado desde los primeros reportes a partir de 1970 hasta la fecha. Esta tabla es muy ambigua para el clínico especialista, puesto que sus valores son demasiado inespecíficos: no define bien lo que se debe considerar razas grandes y pequeñas, los rangos de referencia son muy amplios y no se conoce la metodología ni las medidas utilizadas para obtener los valores reportados $(2,3)$.

Kirk Robert, unos de los internistas más reconocidos a nivel internacional en Medicina Veterinaria, es uno de los autores que reporta esta tabla de referencia, afirma: "Se admite que las determinaciones electrocardiográficas normales y anormales se solapan y que los criterios de normalidad son únicamente orientativos para el clínico. Conforme se vaya disponiendo de más datos estadísticos de los ECG de perros de diferentes razas, conformación, edad y sexo, los datos aquí expuestos precisarán una revisión y se podrá definir con más precisión el termino normal" (2-5).
Uno de los propósitos de este proyecto de investigación y la construcción de la base de datos mencionada, es replantear la organización de la información de estándares cardiacos en caninos, para una definición más precisa del término normal de acuerdo a los parámetros cardiográficos. En tal sentido, se trata de definir lo que se puede considerar normal y anormal, teniendo en cuenta otras variables como por ejemplo peso, sexo, edad, y utilización zootécnica del animal, reduciendo el margen de error en el diagnóstico final.

Los valores de referencia actuales fueron obtenidos sin haber realizado a estos animales otros exámenes previos, como ecocardiografía y radiología, para descartar cardiopatías existentes diferentes a las que se pudieran descartar con el ECG. Entonces, se puede presumir que dentro del total de perros muestreados para construir la tabla de referencia, hubo un porcentaje de perros cardiópatas no detectados por electrocardiografía e incluidos en la base de datos, que pudieron alterar los rangos electrocardiográficos establecidos en la tabla. Sin embargo, esta fué construida por experiencia y consenso entre algunos veterinarios.

Algunos de los interrogantes que se abordaron con la realización de este estudio, fueron: ¿Al realizar exámenes previos como radiografía de tórax y ecocardiografía, se reducirán o ampliaran los rangos de referencia electrocardiográficos?; si se tienen en cuenta variables como edad, raza, sexo, ¿Qué grado de cambios significativos tendremos en los valores de referencia electrocardiográficos?, ¿Qué otras variables podemos tener en cuenta en un ECG que generen nueva información?, ¿Qué otras medidas de apoyo pueden surgir de la utilización conjunta del ECG, ecocardiografía y datos clínicos?

\section{Materiales y métodos}

\section{Aspectos preliminares}

La recolección de la información requiere que se tengan en cuenta ciertos aspectos y condiciones respecto a la población examinada, los instrumentos de 
medida y el espacio físico donde se desarrollan los exámenes. Entre estos, se encuentran los siguientes:

- Realizar la toma de muestras: electrocardiografía, y ecocardiografía en no menos de 100 caninos de diferentes razas, Tablas 1 y 2.

- Establecer las patologías cardiacas existentes y evidentes en cualquiera de las dos pruebas, para descartarlos de la base de datos.

- Organizar la información de los pacientes sin patologías evidentes.

- Construcción de la base de datos.

- Organización de esta información y construcción de nueva información utilizando herramientas tradicionales y otras nuevas propuestas.

Tabla 1: Distribución de las muestras según datos de reseña en los 100 caninos. La cantidad entre paréntesis es el número de caninos de acuerdo a esa característica.

\begin{tabular}{ccc}
\hline Por sexo & Por peso & Por edad \\
\hline Machos (46) & $2-10 \mathrm{Kg}(33)$ & $1-5$ años (53) \\
Hembras (54) & $11-20 \mathrm{Kg}(17)$ & $6-10$ años (37) \\
& $21-30 \mathrm{Kg}(25)$ & $>11$ años (10) \\
& $31 \mathrm{Kg} 0$ más (25) & \\
\hline
\end{tabular}

Tabla 2: Distribución de la población de pacientes según su raza. La cantidad entre paréntesis es el número de caninos de esa raza.

\begin{tabular}{lll}
\hline Labrador (16) & Sharpei (6) & Pitbull (1) \\
French Poodle (14) & Afgano (2) & West Highland T. (1) \\
Schnauzer (7) & Jack Roussel (2) & Chow chow (2) \\
Beagle (5) & Dálmata (1) & Pug (1) \\
Bulldog I. (5) & Akita (2) & Golden R. (5) \\
Pinscher (4) & Maltés (1) & Pastor alemán (2) \\
Basset H. (3) & Yorkshire (1) & Dogo Alemán (1) \\
Weimaraner (3) & Bóxer (3) & Rottweiler (1) \\
Bernés (1) & Criollo (2) & Cruce (1) \\
Scottish T. (3) & Criollo (1) & Pointer A. (1)
\end{tabular}

\section{Procedimientos:}

- Montaje del laboratorio para realizar los dos exámenes lo más simultáneamente posible y en las mejores condiciones técnicas.

- Revisión y organización bibliográfica de los valores estándares actuales considerados normales en caninos en los dos exámenes mencionados.

- Realizar los dos exámenes a no menos de 100 caninos en forma exitosa y desechar aquellos que se consideren de poca calidad, o con datos incompletos.

- Construcción de la base de datos que garantice la veracidad y calidad de la información recolectada.

- Desarrollo de criterios de calificación y clasificación de los resultados para la interpretación y correlación de las medidas de normalidad.

\section{Adquisición de los Registros ECG}

La población examinada corresponde a 102 pacientes caninos sin deficiencias cardiacas de acuerdo a la información obtenida por el examen de ecocardiografía, que se muestrearon en la Clínica Veterinaria "Veteriland" de la ciudad de Bogotá, con ayuda de un equipo de adquisición de señales fisiológicas de la Universidad Antonio Nariño, los cuales tienen características clínicas y físicas heterogéneas. A cada uno se le realizó un examen para adquirir sus datos electrocardiográficos y ecocardiográficos. De esta manera, la base de datos está conformada por las señales de electrocardiografía con sus respectivas medidas, representadas en la duración y la amplitud de las ondas y segmentos de interés, fué así como de los datos que se obtienen en el examen de ecocardiografía para cada paciente.

Los registros ECG fueron adquiridos con la ayuda de un equipo de adquisición de señales fisiológicas marca Biopac Systems. Todo el sistema de adquisición está conformado por los siguientes componentes, Figura 1. 


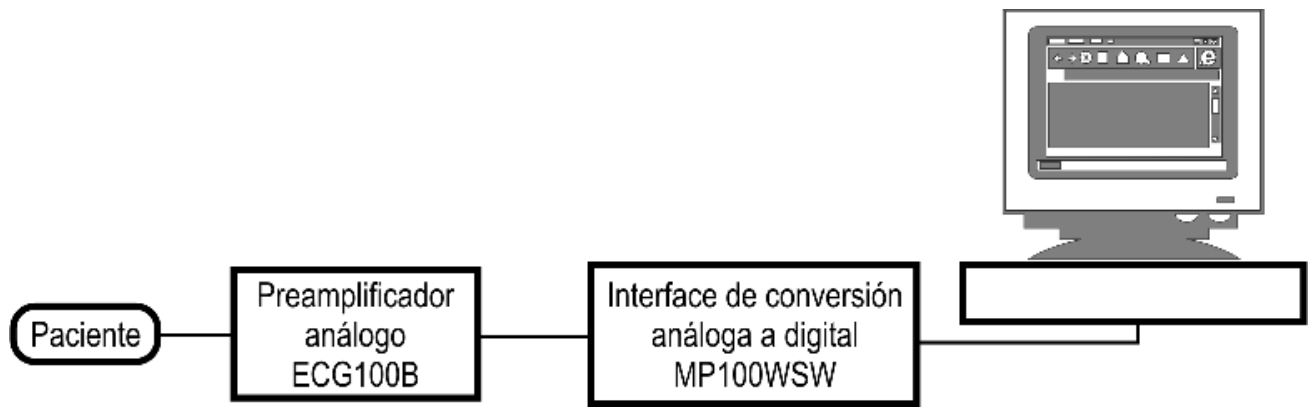

Figura 1: Modelo del sistema de adquisición de señales utilizado

- Computador personal convencional con el programa AcqKnowledge 3.2.3, especializado para investigación, el cual facilita el análisis, visualización y procesamiento de los registros ECG.

- Módulo de adquisición modelo ECG100B, con controles que permiten adecuar la señal antes de ser digitalizada, mediante su respectiva amplificación y atenuación de frecuencias que no hacen parte de la información electrocardiográfica. La señal que recibe el módulo ECG100B proviene de los electrodos ubicados en la superficie del paciente, que luego es enviada en forma analógica, amplificada y filtrada, a un módulo de digitalización. En este punto, el ancho de banda (espectro de componentes de frecuencia) de cada registro ECG puede ser controlado, y para estas medidas se limita a valores entre 1 y $35 \mathrm{~Hz}$. Este filtro permite reducir la contaminación de los datos con tendencias de baja frecuencia y ruido de alta frecuencia que no hacen parte de la información electrocardiográfica. Es válido limitar el ancho de banda de las señales a este rango debido al estado de normalidad de los pacientes examinados y el reposo en el que se encontraban en el instante de la adquisición, considerando que la información de la actividad cardiaca se encuentra entre 1 y $40 \mathrm{~Hz}$.

- Interface MP100WSW, que desempeña funciones de tarjeta de digitalización de la señal, de tal manera que pueda ser interpretada por el computador. En este sentido, el computador debe recibir la señal en forma discreta y no continua, para lo cual cuantifica a sistema binario muestras obtenidas en instantes de tiempo determinados por una tasa de muestreo, que en este caso corresponde a 300 muestras por segundo.

\section{Adquisición de los datos de ecocardiografía}

La ecocardiografía se realizó a través de la ventana paraesternal derecha utilizando un ecógrafo Falco, marca Pie Medical, con software para cardiología. Se realizaron cortes en eje corto y largo, en modo B y M, para el análisis mecánico y funcional del corazón y para la obtención de los valores respectivos para el ventrículo izquierdo, así como los valores de la fracción de acortamiento y eyección del mismo.

\section{Estructura de la base de datos}

Se realizó la organización de los datos en un formato de Microsoft Acces, generando seis tablas con un dato común para todas que corresponde al número de historia clínica. Cada columna es una característica, y las filas representan los pacientes examinados, Figura 2.

La tabla Reseña contiene los datos clínicos básicos de los pacientes, con información sobre nombre, sexo, edad, peso, fecha del examen y raza de cada uno. Las siguientes tres: Datos DI, Datos DII y Datos DIII, contienen algunas características medidas en los registros ECG para cada una de las tres derivaciones bipolares adquiridas. La notación de estas medidas, ubicadas en cada columna de las tablas, se representa en la Tabla 3 y la Figura 3.

Así mismo, se incluyen columnas en las tablas para indicar las medidas de amplitud y duración de las ondas $\mathrm{R}^{\prime}$ en caso de que existan (ondas $\mathrm{R}$ que se 


\section{Dicrosoft Access - [VETUAN : Base de datos (Formato de archivo de Access 2000)]}

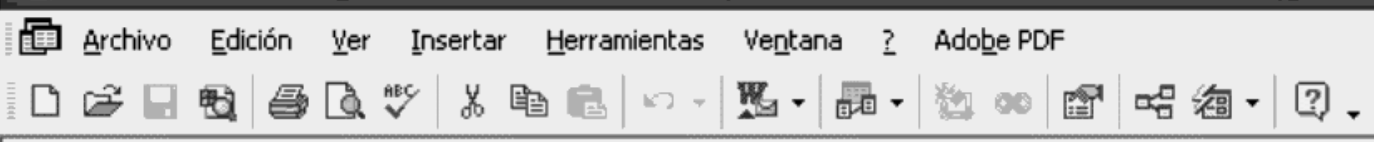

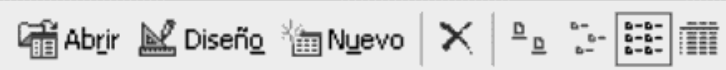

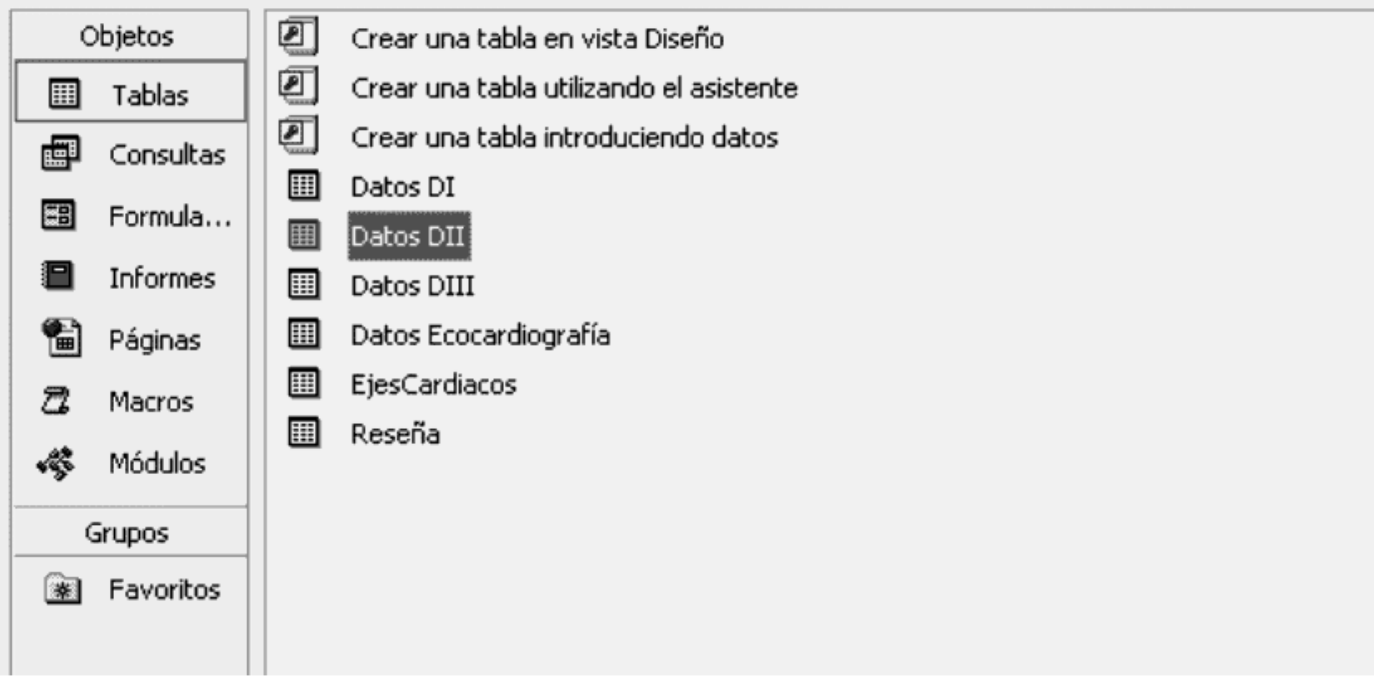

Figura 2. Vista principal de la distribución en tablas de la base de datos VETUAN_I.

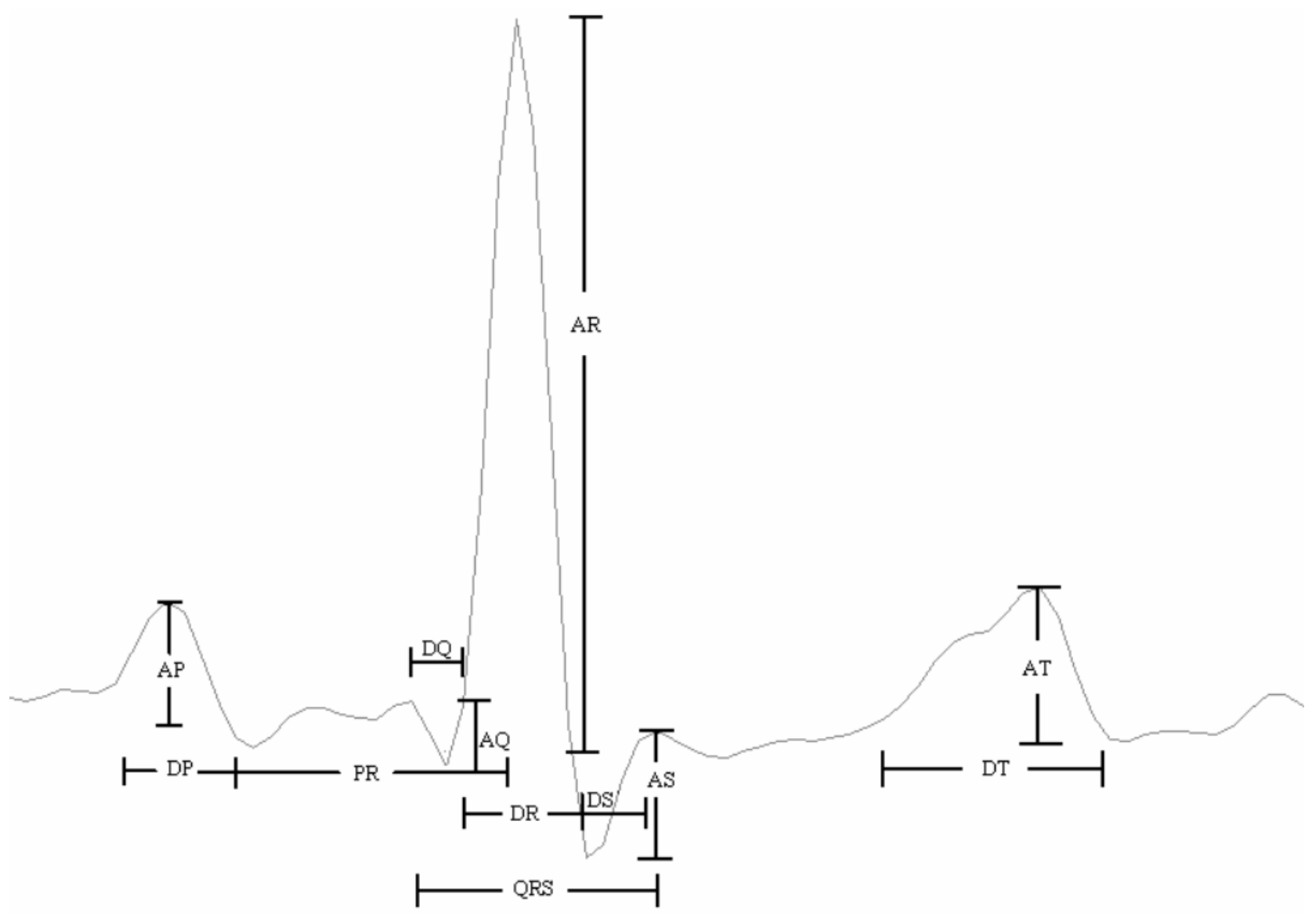

Figura 3. Representación de las características medidas. 


\begin{tabular}{cl}
\hline Notación & \multicolumn{1}{c}{ Significado } \\
\hline \hline AP & Amplitud onda P (mV) \\
DP & Duración onda P \\
DR & Duración del segmento PR (s) \\
AQ & Duración de la onda Q (s) \\
DR & Amplitud de la onda Q (mV) \\
ARprima & Duración onda R (s) \\
& Amplitud de onda R si existe \\
DRprima & Duración de onda R si existe (s) \\
AR & Amplitud onda R (mV) \\
DS & Duración onda S (s) \\
AS & Amplitud onda S (mV) \\
QRS & Duración complejo QRS (s) \\
AT & Amplitud onda T (mV) \\
DT & Duración onda T (s) \\
Morfología onda P y T & 0-positivo, 1-negativo, 2-bifásico
\end{tabular}

Tabla 3. Notación de las características medidas en cada una de las derivaciones bipolares de las señales ECG. repiten inmediatamente después de la presencia de las ondas R principales), como se observa en la Figura 4. Todos estos datos fueron estimados de forma visual con ayuda del programa AcqKnowledge 3.2.3., sobre los ciclos cardiacos que a simple vista resultaban ser los más representativos. Cabe anotar que el programa AcqKnowledge 3.2.3. incluye una herramienta que determina estas medidas, cuando de forma manual se selecciona la onda o el segmento de interés a partir del ciclo cardiaco más representativo, Figura 5.

La tabla Ejes Cardiacos contiene los datos correspondientes a los ejes cardiacos para cada paciente.

\section{Análisis de los datos}

La información obtenida se organizó de modo que pueda ser analizada de acuerdo al sexo de los pacientes con respecto a su peso, tanto para cada una de las derivaciones electrocardiográficas (DI DII y DIII), como para los datos de ecocardiografía, los cuales no incluyen en el análisis las medidas de las ondas R', ni de morfología para las ondas P y T (características identificadas como ARprima, DRprima,

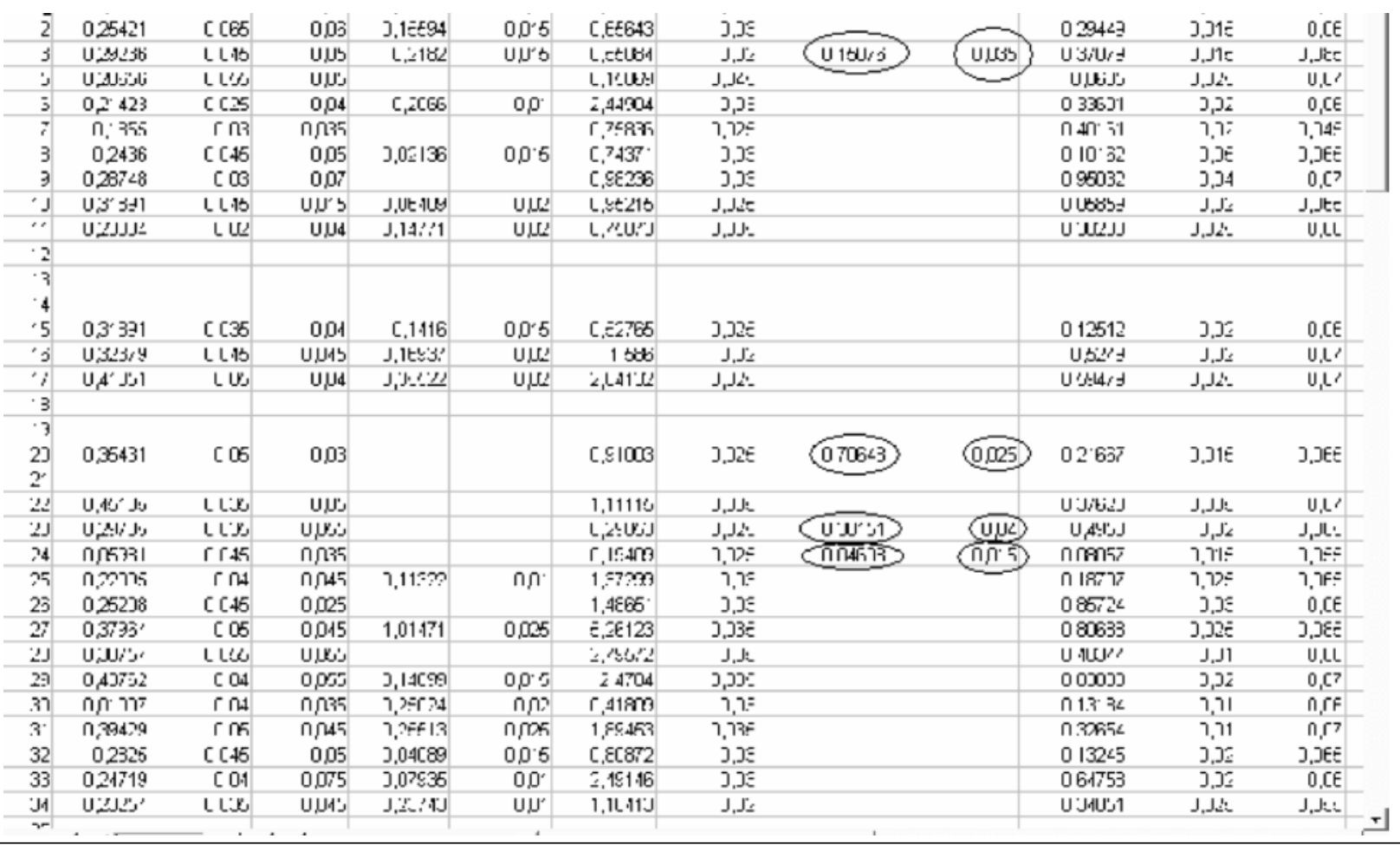

Figura 4: Vista de una de las tablas de medidas de ECG en la base de datos VETUAN_I. Los óvalos indican que esos pacientes presentaron ondas R', y de ahí sus medidas de amplitud y duración. 


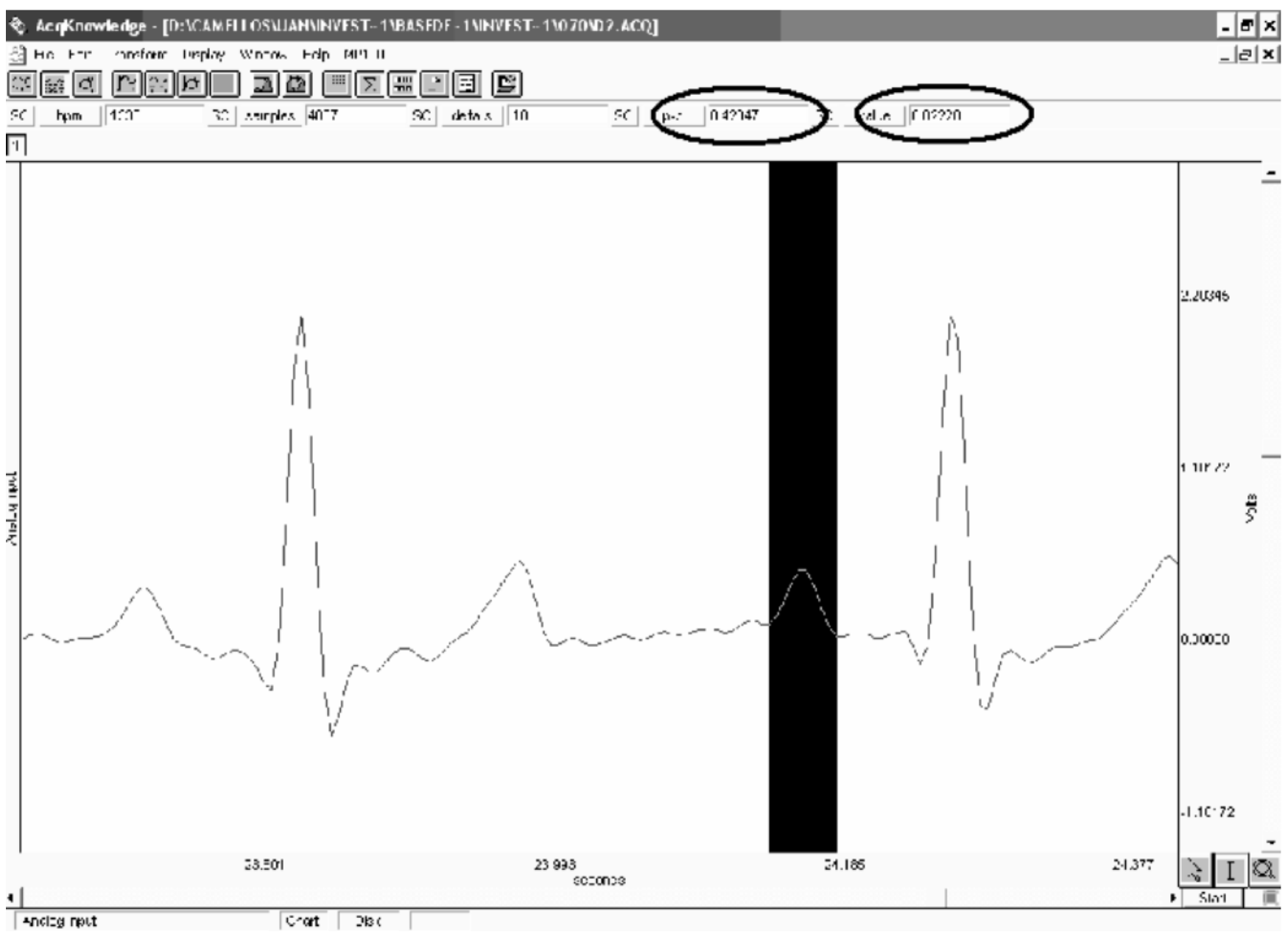

Figura 5: Medición de duración y amplitud para una onda P. Los óvalos indican las secciones del programa AcqKnowledge 3.2.3.

y Morfología onda P y T en la Tabla 3), ya que las ondas R' se presentan muy escasamente en la población examinada, mientras que los datos de morfología son de tipo cualitativo.

Los anexos presentados contienen la distribución mencionada con los promedios, así como por los valores máximos y mínimos de las medidas analizadas. Los promedios están compuestos por la media $(\bar{x})$ calculada mediante la expresión, y por la desviación estándar $\left(\frac{1}{n} \sum x_{n}\right)$ calculada mediante la raíz cuadrada de $\frac{n \sum x_{n}^{2}-\left(\sum x_{n}\right)^{2}}{n(n-1)}$, donde $\boldsymbol{n}$ corresponde al número de muestras. De igual forma, se indica la cantidad de pacientes en cada uno de los grupos por peso, ya que no todos los grupos tienen la misma cantidad de registros.

\section{Resultados}

El Anexo A presenta la tabla de referencia de medidas electrocardiográficas para pacientes caninos sin anomalías cardiacas residentes en la ciudad de Bogotá. El Anexo B presenta las gráficas para los valores de la media en las medidas de electrocardiografía, según el sexo de los pacientes y agrupados por peso. Los números entre paréntesis en las leyendas de cada gráfica representan la cantidad de pacientes al que corresponde el respectivo grupo dividido por peso.

De acuerdo a la inspección visual de las gráficas, se puede observar que la medida de amplitud de la onda $\mathrm{R}$ en todas las derivaciones puede ser la característica más discriminante entre pacientes de diferente peso, lo cual concuerda con el tamaño del músculo cardiaco conforme aumenta el peso de los pacientes. Adicionalmente, resulta difícil establecer un criterio de separabilidad entre 
Anexo A DERIVACIÓN DI MACHOS

\begin{tabular}{|c|c|c|c|}
\hline \multicolumn{4}{|c|}{$<10 \mathrm{Kg}$ (13 pacientes) } \\
\hline & Promedio & Máximo & Mínimo \\
\hline$A P$ & $0,370 \pm 0,144$ & 0,696 & 0,169 \\
\hline$D P$ & $0,033 \pm 0,013$ & 0,05 & 0,015 \\
\hline$P R$ & $0,054 \pm 0,014$ & 0,075 & 0,03 \\
\hline$A Q$ & $0,220 \pm 0,210$ & 0,524 & 0,059 \\
\hline$D Q$ & $0,018 \pm 0,009$ & 0,025 & 0,005 \\
\hline$A R$ & $0,799 \pm 0,455$ & 1,993 & 0,365 \\
\hline$D R$ & $0,027 \pm 0,007$ & 0,04 & 0,015 \\
\hline AS & $0,152 \pm 0,087$ & 0,268 & 0,038 \\
\hline DS & $0,023 \pm 0,013$ & 0,05 & 0,005 \\
\hline QRS & $0,047 \pm 0,020$ & 0,075 & 0,02 \\
\hline ST & $0,053 \pm 0,016$ & 0,075 & 0,02 \\
\hline AT & $0,353 \pm 0,189$ & 0,721 & 0,057 \\
\hline DT & $0,044 \pm 0,021$ & 0,075 & 0,015 \\
\hline \multicolumn{4}{|c|}{$30-39.9 \mathrm{Kg}$ (15 pacientes) } \\
\hline & Promedio & Máximo & Minimo \\
\hline$A P$ & $0,273 \pm 0,080$ & 0,425 & 0,106 \\
\hline$D P$ & $0,040 \pm 0,010$ & 0,055 & 0,02 \\
\hline$P R$ & $0,071 \pm 0,021$ & 0,1 & 0,035 \\
\hline$A Q$ & $0,149 \pm 0,093$ & 0,276 & 0,051 \\
\hline$D Q$ & $0,024 \pm 0,011$ & 0,04 & 0,01 \\
\hline$A R$ & $0,731 \pm 0,387$ & 1,219 & 0,133 \\
\hline$D R$ & $0,031 \pm 0,010$ & 0,055 & 0,015 \\
\hline AS & $0,175 \pm 0,112$ & 0,324 & 0,03 \\
\hline$D S$ & $0,024 \pm 0,010$ & 0,05 & 0,01 \\
\hline QRS & $0,057 \pm 0,013$ & 0,075 & 0,03 \\
\hline ST & $0,068 \pm 0,014$ & 0,09 & 0,045 \\
\hline$A T$ & $0,348 \pm 0,151$ & 0,603 & 0,155 \\
\hline DT & $0,050 \pm 0,016$ & 0,08 & 0,025 \\
\hline
\end{tabular}

\begin{tabular}{|c|c|c|c|}
\hline \multicolumn{4}{|c|}{ DERIVACION DI MACHOS } \\
\hline \multicolumn{4}{|c|}{$10-19.9 \mathrm{Kg}$ (5 pacientes) } \\
\hline & Promedio & Máximo & Mínimo \\
\hline$A P$ & $0,281 \pm 0,193$ & 0,562 & 0,119 \\
\hline$D P$ & $0,035 \pm 0,018$ & 0,055 & 0,02 \\
\hline$P R$ & $0,061 \pm 0,027$ & 0,095 & 0,04 \\
\hline$A Q$ & $0,204 \pm 0,014$ & 0,214 & 0,194 \\
\hline$D Q$ & $0,018 \pm 0,004$ & 0,02 & 0,015 \\
\hline$A R$ & $1,220 \pm 0,627$ & 1,994 & 0,575 \\
\hline$D R$ & $0,030 \pm 0,004$ & 0,035 & 0,025 \\
\hline AS & $0,490 \pm 0,562$ & 0,888 & 0,093 \\
\hline DS & $0,020 \pm 0,007$ & 0,025 & 0,015 \\
\hline QRS & $0,048 \pm 0,015$ & 0,06 & 0,03 \\
\hline ST & $0,083 \pm 0,019$ & 0,105 & 0,06 \\
\hline$A T$ & $0,251 \pm 0,122$ & 0,375 & 0,132 \\
\hline DT & $0,046 \pm 0,022$ & 0,07 & 0,025 \\
\hline \multicolumn{4}{|c|}{$40-49.9 \mathrm{Kg}$ ( 2 pacientes) } \\
\hline & Promedio & Máximo & Mínimo \\
\hline$A P$ & $0,297 \pm 0,269$ & 0,487 & 0,106 \\
\hline$D P$ & $0,043 \pm 0,011$ & 0,05 & 0,035 \\
\hline$P R$ & $0,060 \pm 0,007$ & 0,065 & 0,055 \\
\hline$A Q$ & $0,207 \pm 0,173$ & 0,329 & 0,084 \\
\hline$D Q$ & $0,018 \pm 0,004$ & 0,02 & 0,015 \\
\hline$A R$ & $1,091 \pm 0,014$ & 1,1 & 1,081 \\
\hline$D R$ & $0,033 \pm 0,004$ & 0,035 & 0,03 \\
\hline AS & $0,119 \pm 0,048$ & 0,153 & 0,085 \\
\hline$D S$ & $0,020 \pm 0,000$ & 0,02 & 0,02 \\
\hline QRS & $0,070 \pm 0,000$ & 0,07 & 0,07 \\
\hline$S T$ & $0,068 \pm 0,032$ & 0,09 & 0,045 \\
\hline AT & $0,243 \pm 0,259$ & 0,427 & 0,06 \\
\hline$D T$ & $0,073 \pm 0,004$ & 0,075 & 0,07 \\
\hline
\end{tabular}

\begin{tabular}{c|c|c|c}
\hline \multicolumn{4}{|c}{$20-29.9 \mathrm{Kg}$ (10 pacientes) } \\
\hline \hline & Promedio & Máximo & Mínimo \\
\hline \hline AP & $0,313 \pm 0,159$ & 0,58 & 0,096 \\
DP & $0,044 \pm 0,018$ & 0,065 & 0,01 \\
$P R$ & $0,051 \pm 0,028$ & 0,105 & 0,015 \\
AQ & $0,067 \pm 0,044$ & 0,11 & 0,023 \\
DQ & $0,010 \pm 0,000$ & 0,01 & 0,01 \\
AR & $1,053 \pm 0,610$ & 2,037 & 0,121 \\
DR & $0,036 \pm 0,011$ & 0,055 & 0,02 \\
AS & $0,303 \pm 0,224$ & 0,664 & 0,002 \\
DS & $0,024 \pm 0,007$ & 0,035 & 0,015 \\
QRS & $0,120 \pm 0,158$ & 0,512 & 0,055 \\
ST & $0,065 \pm 0,032$ & 0,115 & 0,025 \\
AT & $0,305 \pm 0,207$ & 0,612 & 0,1 \\
DT & $0,052 \pm 0,020$ & 0,075 & 0,02 \\
\hline
\end{tabular}

Para $>50 \mathrm{Kg}$ no hay datos

DERIVACIÓN DI HEMBRAS

\begin{tabular}{c|c|c|c}
\hline \multicolumn{4}{|c}{$<10 \mathrm{Kg}(20$ pacientes $)$} \\
\hline & Promedio & Máximo & Mínimo \\
\hline \hline$A P$ & $0,378 \pm 0,147$ & 0,808 & 0,203 \\
$D P$ & $0,037 \pm 0,010$ & 0,05 & 0,02 \\
$P R$ & $0,050 \pm 0,016$ & 0,095 & 0,03 \\
$A Q$ & $0,119 \pm 0,059$ & 0,176 & 0,032 \\
$D Q$ & $0,017 \pm 0,005$ & 0,025 & 0,01 \\
$A R$ & $0,970 \pm 0,399$ & 1,934 & 0,597 \\
$D R$ & $0,030 \pm 0,006$ & 0,04 & 0,02 \\
$A S$ & $0,294 \pm 0,181$ & 0,76 & 0,023 \\
$D S$ & $0,019 \pm 0,007$ & 0,03 & 0,005 \\
QRS & $0,056 \pm 0,013$ & 0,07 & 0,03 \\
ST & $0,050 \pm 0,022$ & 0,095 & 0,02 \\
AT & $0,341 \pm 0,186$ & 0,796 & 0,13 \\
$D T$ & $0,051 \pm 0,010$ & 0,06 & 0,02 \\
\hline
\end{tabular}

\begin{tabular}{c|c|c|c}
\hline \multicolumn{4}{|c|}{$10-19.9 \mathrm{Kg}$ (9 pacientes) } \\
\hline & Promedio & Máximo & Mínimo \\
\hline \hline AP & $0,289 \pm 0,136$ & 0,575 & 0,178 \\
DP & $0,031 \pm 0,015$ & 0,05 & 0,015 \\
PR & $0,053 \pm 0,023$ & 0,105 & 0,035 \\
AQ & $0,227 \pm 0,182$ & 0,564 & 0,059 \\
DQ & $0,012 \pm 0,006$ & 0,02 & 0,005 \\
AR & $0,612 \pm 0,539$ & 1,978 & 0,199 \\
DR & $0,026 \pm 0,008$ & 0,035 & 0,01 \\
AS & $0,191 \pm 0,281$ & 0,868 & 0,003 \\
DS & $0,022 \pm 0,011$ & 0,03 & 0,005 \\
QRS & $0,051 \pm 0,020$ & 0,075 & 0,02 \\
ST & $0,058 \pm 0,031$ & 0,13 & 0,03 \\
AT & $0,295 \pm 0,092$ & 0,413 & 0,122 \\
DT & $0,048 \pm 0,010$ & 0,065 & 0,035 \\
\hline
\end{tabular}

\begin{tabular}{|c|c|c|c|}
\hline \multicolumn{4}{|c|}{$20-29.9 \mathrm{Kg}$ (15 pacientes) } \\
\hline & Promedio & Máximo & Mínimo \\
\hline$A P$ & $0,256 \pm 0,127$ & 0,657 & 0,143 \\
\hline$D P$ & $0,034 \pm 0,015$ & 0,06 & 0,015 \\
\hline$P R$ & $0,066 \pm 0,027$ & 0,12 & 0,03 \\
\hline$A Q$ & $0,391 \pm 0,415$ & 1,09 & 0,048 \\
\hline$D Q$ & $0,012 \pm 0,005$ & 0,02 & 0,005 \\
\hline$A R$ & $1,169 \pm 1,011$ & 3,913 & 0,145 \\
\hline$D R$ & $0,028 \pm 0,009$ & 0,04 & 0,015 \\
\hline AS & $0,333 \pm 0,246$ & 0,777 & 0,125 \\
\hline$D S$ & $0,023 \pm 0,005$ & 0,03 & 0,015 \\
\hline QRS & $0,046 \pm 0,012$ & 0,07 & 0,03 \\
\hline ST & $0,068 \pm 0,026$ & 0,12 & 0,04 \\
\hline$A T$ & $0,319 \pm 0,179$ & 0,564 & 0,143 \\
\hline DT & $0,051 \pm 0,012$ & 0,07 & 0,03 \\
\hline \multicolumn{4}{|c|}{$40-49.9 \mathrm{Kg}$ (2 pacientes) } \\
\hline & Promedio & Máximo & Minimo \\
\hline$A P$ & $0,320 \pm 0,055$ & 0,359 & 0,281 \\
\hline$D P$ & $0,045 \pm 0,007$ & 0,05 & 0,04 \\
\hline$P R$ & $0,070 \pm 0,035$ & 0,095 & 0,045 \\
\hline$A Q$ & -- & --- & -- \\
\hline$D Q$ & $-\mathbf{n}^{-}$ & --- & $\cdots$ \\
\hline$A R$ & $1,063 \pm 0,133$ & 1,157 & 0,968 \\
\hline$D R$ & $0,035 \pm 0,014$ & 0,045 & 0,025 \\
\hline AS & $0,280 \pm 0,256$ & 0.461 & 0,099 \\
\hline$D S$ & $0,018 \pm 0,004$ & 0,02 & 0,015 \\
\hline QRS & $0,053 \pm 0,011$ & 0,06 & 0,045 \\
\hline$S T$ & $0,080 \pm 0,021$ & 0,095 & 0,065 \\
\hline$A T$ & $0,311 \pm 0,123$ & 0,398 & 0,223 \\
\hline DT & $0,053 \pm 0,018$ & 0,065 & 0,04 \\
\hline
\end{tabular}


Anexo A

DERIVACIÓN DII MACHOS

\begin{tabular}{|c|c|c|c|}
\hline \multicolumn{4}{|c|}{$<10 \mathrm{Kg}$ (13 pacientes) } \\
\hline & Promedio & Máximo & Mínimo \\
\hline$A P$ & $0,373 \pm 0,172$ & 0,641 & 0,148 \\
\hline$D P$ & $0,035 \pm 0,011$ & 0,05 & 0,02 \\
\hline$P R$ & $0,060 \pm 0,021$ & 0,095 & 0,03 \\
\hline$A Q$ & $0,231 \pm 0,199$ & 0,547 & 0,045 \\
\hline$D Q$ & $0,012 \pm 0,003$ & 0,015 & 0,01 \\
\hline$A R$ & $1,447 \pm 1,132$ & 3,993 & 0,274 \\
\hline$D R$ & $0,029 \pm 0,009$ & $0,0<5$ & 0,01 \\
\hline AS & $0,505 \pm 0,235$ & 0,903 & 0,175 \\
\hline DS & $0,023 \pm 0,014$ & 0,05 & 0,01 \\
\hline QRS & $0,051 \pm 0,018$ & 0,08 & 0,02 \\
\hline ST & $0,064 \pm 0,024$ & 0,115 & 0,02 \\
\hline AT & $0,439 \pm 0,288$ & 1,012 & 0,144 \\
\hline DT & $0,052 \pm 0,021$ & 0,1 & 0,03 \\
\hline \multicolumn{4}{|c|}{$30-39.9 \mathrm{Kg}$ (15 pacientes) } \\
\hline & Promedio & Máximo & Mínimo \\
\hline$A P$ & $0,426 \pm 0,180$ & 0,79 & 0,173 \\
\hline$D P$ & $0,047 \pm 0,010$ & 0,07 & 0,03 \\
\hline$P R$ & $0,064 \pm 0,026$ & 0,115 & 0,015 \\
\hline$A Q$ & $0,346 \pm 0,250$ & 0,892 & 0,049 \\
\hline$D Q$ & $0,024 \pm 0,010$ & 0,05 & 0,015 \\
\hline$A R$ & $1,725 \pm 0,764$ & 3,17 & 0,493 \\
\hline$D R$ & $0,031 \pm 0,005$ & 0,04 & 0,02 \\
\hline AS & $0,367 \pm 0,317$ & 1,098 & 0,029 \\
\hline$D S$ & $0,018 \pm 0,005$ & 0,03 & 0,01 \\
\hline QRS & $0,059 \pm 0,017$ & 0,075 & 0,03 \\
\hline ST & $0,065 \pm 0,022$ & 0,105 & 0,02 \\
\hline AT & $0,501 \pm 0,232$ & 0,799 & 0,05 \\
\hline DT & $0,055 \pm 0,016$ & 0,08 & 0,02 \\
\hline
\end{tabular}

\begin{tabular}{|c|c|c|c|}
\hline \multicolumn{4}{|c|}{$10-19.9 \mathrm{Kg}$ ( 5 pacientes) } \\
\hline & Promedio & Máximo & Minimo \\
\hline$A P$ & $0,462 \pm 0,090$ & 0,569 & 0,35 \\
\hline$D P$ & $0,044 \pm 0,019$ & 0,06 & 0,025 \\
\hline$P R$ & $0,061 \pm 0,043$ & 0,105 & 0,015 \\
\hline$A Q$ & $0,183 \pm 0,137$ & 0,326 & 0,052 \\
\hline$D Q$ & $0,013 \pm 0,003$ & 0,015 & 0,01 \\
\hline$A R$ & $1,953 \pm 0,139$ & 2,16 & 1,867 \\
\hline$D R$ & $0,028 \pm 0,005$ & 0,03 & 0,02 \\
\hline AS & $0,427 \pm 0,474$ & 1,107 & 0,041 \\
\hline DS & $0,015 \pm 0,008$ & 0,025 & 0,005 \\
\hline QRS & $0,053 \pm 0,006$ & 0,06 & 0,045 \\
\hline$S T$ & $0,066 \pm 0,021$ & 0,09 & 0,04 \\
\hline AT & $0,259 \pm 0,146$ & 0,369 & 0,045 \\
\hline$D T$ & $0,064 \pm 0,019$ & 0,085 & 0,045 \\
\hline \multicolumn{4}{|c|}{$40-49,9 \mathrm{Kg}$ (3 pacientes) } \\
\hline & Promedio & Máximo & Minimo \\
\hline$A P$ & $0,373 \pm 0,164$ & 0,489 & 0,257 \\
\hline$D P$ & $0,055 \pm 0,007$ & 0,06 & 0,05 \\
\hline$P R$ & $0,043 \pm 0,004$ & $0,0<5$ & 0,04 \\
\hline$A Q$ & $0,316 \pm 0,442$ & 0,629 & 0,004 \\
\hline$D Q$ & $0,020 \pm 0,007$ & 0,025 & 0,015 \\
\hline$A R$ & $1,433 \pm 0,161$ & 1,547 & 1,319 \\
\hline$D R$ & $0,028 \pm 0,004$ & 0,03 & 0,025 \\
\hline AS & $0,639 \pm 0,450$ & 0,957 & 0,321 \\
\hline$D S$ & $0,020 \pm 0,000$ & 0,02 & 0,02 \\
\hline QRS & $0,068 \pm 0,004$ & 0,07 & 0,065 \\
\hline ST & $0,080 \pm 0,021$ & 0,095 & 0,065 \\
\hline AT & $0,409 \pm 0,302$ & 0,622 & 0,195 \\
\hline$D T$ & $0,055 \pm 0,007$ & 0,06 & 0,05 \\
\hline
\end{tabular}

\begin{tabular}{c|c|c|c}
\hline \multicolumn{4}{|c}{$20-29.9 \mathrm{Kg}$ (10 pacientes) } \\
\hline & Promedio & Máximo & Mínimo \\
\hline \hline$A P$ & $0,404 \pm 0,231$ & 0,755 & 0,044 \\
$D P$ & $0,0<3 \pm 0,012$ & 0,065 & 0,03 \\
$P R$ & $0,071 \pm 0,024$ & 0,105 & 0,045 \\
$A Q$ & $0,264 \pm 0,230$ & 0,64 & 0,057 \\
$D Q$ & $0,018 \pm 0,008$ & 0,03 & 0,01 \\
$A R$ & $2,207 \pm 1,221$ & 5,283 & 1,11 \\
$D R$ & $0,034 \pm 0,007$ & 0,05 & 0,025 \\
$A S$ & $0,589 \pm 0,452$ & 1,533 & 0,162 \\
$D S$ & $0,023 \pm 0,005$ & 0,03 & 0,02 \\
$Q R S$ & $0,061 \pm 0,019$ & 0,075 & 0,02 \\
$S T$ & $0,071 \pm 0,018$ & 0,105 & 0,045 \\
$A T$ & $0,479 \pm 0,360$ & 1,14 & 0,073 \\
$D T$ & $0,059 \pm 0,010$ & 0,075 & 0,05 \\
\hline
\end{tabular}

\section{DERIVACIÓN DII HEMBRAS}

\begin{tabular}{|c|c|c|c|}
\hline \multicolumn{4}{|c|}{$<10 \mathrm{Kg}$ (20 pacientes) } \\
\hline & Promedio & Máximo & Mínimo \\
\hline$A P$ & $0,431 \pm 0,164$ & 0,653 & 0,148 \\
\hline$D P$ & $0,039 \pm 0,012$ & 0,07 & 0,02 \\
\hline$P R$ & $0,046 \pm 0,023$ & 0,115 & 0,015 \\
\hline$A Q$ & $0,236 \pm 0,112$ & 0,445 & 0,092 \\
\hline$D Q$ & $0,016 \pm 0,004$ & 0,025 & 0,01 \\
\hline$A R$ & $1,686 \pm 0,828$ & $3,6<3$ & 0,673 \\
\hline$D R$ & $0,029 \pm 0,008$ & 0,045 & 0,02 \\
\hline AS & $0,559 \pm 0,301$ & 1,527 & 0,065 \\
\hline DS & $0,024 \pm 0,013$ & 0,05 & 0,01 \\
\hline QRS & $0,059 \pm 0,017$ & 0,08 & 0,03 \\
\hline ST & $0,058 \pm 0,023$ & 0,115 & 0,02 \\
\hline AT & $0,420 \pm 0,262$ & 1,189 & 0,077 \\
\hline DT & $0,051 \pm 0,009$ & 0,065 & 0,03 \\
\hline \multicolumn{4}{|c|}{$30-39.8 \mathrm{Kg}$ (6 pacientes) } \\
\hline & Promedio & Máximo & Minimo \\
\hline$A P$ & $0,284 \pm 0,194$ & 0,532 & 0,058 \\
\hline$D P$ & $0,047 \pm 0,014$ & 0,06 & 0,03 \\
\hline$P R$ & $0,058 \pm 0,025$ & 0,095 & 0,04 \\
\hline$A Q$ & $0,498 \pm 0,400$ & 0,997 & 0,12 \\
\hline$D Q$ & $0,021 \pm 0,004$ & 0,025 & 0,015 \\
\hline$A R$ & $2,215 \pm 0,710$ & 3,559 & 1,425 \\
\hline$D R$ & $0,027 \pm 0,006$ & 0,035 & 0,02 \\
\hline AS & $0,572 \pm 0,332$ & 0,948 & 0,086 \\
\hline$D S$ & $0,020 \pm 0,007$ & 0,03 & 0,01 \\
\hline QRS & $0,068 \pm 0,010$ & 0,075 & 0,05 \\
\hline ST & $0,078 \pm 0,018$ & 0,1 & 0,06 \\
\hline AT & $0,382 \pm 0,153$ & 0,53 & 0,083 \\
\hline DT & $0,052 \pm 0,010$ & 0,065 & 0,04 \\
\hline
\end{tabular}

\begin{tabular}{c|c|c|c}
\hline \multicolumn{4}{|c}{$10-19.9 \mathrm{Kg}$ (9 pacientes) } \\
\hline & Promedio & Máximo & Mínimo \\
\hline \hline AP & $0,407 \pm 0,115$ & 0,556 & 0,275 \\
DP & $0,040 \pm 0,012$ & 0,06 & 0,02 \\
PR & $0,065 \pm 0,035$ & 0,125 & 0,035 \\
AQ & $0,159 \pm 0,059$ & 0,201 & 0,091 \\
DQ & $0,015 \pm 0,000$ & 0,015 & 0,015 \\
AR & $1,457 \pm 1,104$ & 3,993 & 0,6 \\
DR & $0,026 \pm 0,005$ & 0,03 & 0,02 \\
AS & $0,301 \pm 0,308$ & 0,838 & 0,003 \\
DS & $0,020 \pm 0,008$ & 0,03 & 0,01 \\
QRS & $0,051 \pm 0,016$ & 0,07 & 0,03 \\
ST & $0,061 \pm 0,027$ & 0,115 & 0,04 \\
AT & $0,361 \pm 0,239$ & 0,812 & 0,046 \\
DT & $0,061 \pm 0,025$ & 0,115 & 0,04 \\
\hline
\end{tabular}

\begin{tabular}{|c|c|c|c|}
\hline \multicolumn{4}{|c|}{$20-29.9 \mathrm{Kg}$ (15 pacientes) } \\
\hline & Promedio & Máximo & Minimo \\
\hline$A P$ & $0,370 \pm 0,115$ & 0,588 & 0,256 \\
\hline$D P$ & $0,036 \pm 0,010$ & 0,05 & 0,02 \\
\hline$P R$ & $0,068 \pm 0,035$ & 0,137 & 0,03 \\
\hline$A Q$ & $0,500 \pm 0,656$ & 1,98 & $0,0<9$ \\
\hline$D Q$ & $0,017 \pm 0,007$ & 0,03 & 0,01 \\
\hline$A R$ & $1,822 \pm 1,223$ & 4,284 & 0,499 \\
\hline$D R$ & $0,062 \pm 0,117$ & 0,45 & 0,02 \\
\hline AS & $0,366 \pm 0,320$ & 1,016 & 0,093 \\
\hline$D S$ & $0,018 \pm 0,004$ & 0,02 & 0,01 \\
\hline QRS & $0,053 \pm 0,014$ & 0,075 & 0,03 \\
\hline ST & $0,068 \pm 0,019$ & 0,105 & 0,04 \\
\hline$A T$ & $0,313 \pm 0,168$ & 0,731 & 0,094 \\
\hline DT & $0,046 \pm 0,016$ & 0,075 & 0,02 \\
\hline \multicolumn{4}{|c|}{$50 \mathrm{Kg}(1$ oaciente $)$} \\
\hline & Promedio & Máximo & Minimo \\
\hline$A P$ & $0,446 \pm 0,079$ & 0,503 & 0,39 \\
\hline$D P$ & $0,055 \pm 0,014$ & 0,065 & 0,045 \\
\hline$P R$ & $0,055 \pm 0,014$ & 0,065 & 0,045 \\
\hline$A Q$ & --- & -..-- & -..- \\
\hline$D Q$ & ---- & -.-- & -.-- \\
\hline$A R$ & $1,629 \pm 0,167$ & 1,748 & 1,511 \\
\hline$D R$ & $0,038 \pm 0,011$ & 0,045 & 0,03 \\
\hline AS & $0,813 \pm 0,302$ & 1,026 & 0,599 \\
\hline$D S$ & $0,023 \pm 0,004$ & 0,025 & 0,02 \\
\hline QRS & $0,058 \pm 0,004$ & 0,06 & 0,055 \\
\hline ST & $0,055 \pm 0,021$ & 0,07 & 0,04 \\
\hline$A T$ & $0,506 \pm 0,076$ & 0,56 & 0,452 \\
\hline$D T$ & $0,060 \pm 0,007$ & 0,065 & 0,055 \\
\hline
\end{tabular}

www.unicolmayor.edu.co 
Anexo A

DERIVACIÓN DIII MACHOS

\begin{tabular}{c|c|c|c}
\hline \multicolumn{4}{|c}{$<10 \mathrm{Kg}(13$ pacientes) } \\
\hline & Promedio & Máximo & Minimo \\
\hline \hline AP & $0,264 \pm 0,105$ & 0,388 & 0,054 \\
$D P$ & $0,028 \pm 0,013$ & 0,045 & 0,01 \\
$P R$ & $0,054 \pm 0,019$ & 0,08 & 0,035 \\
$A Q$ & $0,219 \pm 0,201$ & 0,614 & 0,027 \\
$D Q$ & $0,013 \pm 0,006$ & 0,02 & 0,005 \\
$A R$ & $0,746 \pm 0,698$ & 2,449 & 0,211 \\
$D R$ & $0,024 \pm 0,009$ & 0,035 & 0,01 \\
AS & $0,295 \pm 0,147$ & 0,461 & 0,049 \\
$D S$ & $0,023 \pm 0,012$ & 0,045 & 0,005 \\
QRS & $0,044 \pm 0,017$ & 0,08 & 0,02 \\
ST & $0,061 \pm 0,019$ & 0,095 & 0,03 \\
AT & $0,401 \pm 0,237$ & 0,748 & 0,138 \\
$D T$ & $0,064 \pm 0,035$ & 0,145 & 0,035 \\
\hline
\end{tabular}

\begin{tabular}{|c|c|c|c|}
\hline \multicolumn{4}{|c|}{$10-19.9 \mathrm{Kg}$ (5 pacientes) } \\
\hline & Promedio & Máximo & Minimo \\
\hline$A P$ & $0,290 \pm 0,181$ & 0,441 & 0.089 \\
\hline$D P$ & $0,030 \pm 0,010$ & 0,04 & 0,02 \\
\hline$P R$ & $0,073 \pm 0,039$ & 0,105 & 0,03 \\
\hline$A Q$ & $0,088 \pm 0,006$ & 0,092 & 0.085 \\
\hline$D Q$ & $0,015 \pm 0,007$ & 0,02 & 0,01 \\
\hline$A R$ & $0,838 \pm 0,448$ & 1,195 & 0.336 \\
\hline$D R$ & $0,023 \pm 0,006$ & 0,03 & 0,02 \\
\hline AS & $0,416 \pm 0,285$ & 0,618 & 0.215 \\
\hline$D S$ & $0,020 \pm 0,000$ & 0,02 & 0,02 \\
\hline QRS & $0,047 \pm 0,012$ & 0,06 & 0,04 \\
\hline ST & $0,083 \pm 0,023$ & 0,105 & 0,06 \\
\hline AT & $0,289 \pm 0,107$ & 0,408 & 0.2 \\
\hline$D T$ & $0,125 \pm 0,134$ & 0,28 & 0.045 \\
\hline \multicolumn{4}{|c|}{$40-49,9 \mathrm{Kg}$ (3 pacientes) } \\
\hline & Promedio & Máximo & Minimo \\
\hline$A P$ & $0,345 \pm 0,113$ & 0,425 & 0,265 \\
\hline$D P$ & $0,048 \pm 0,011$ & 0,055 & 0,04 \\
\hline$P R$ & $0,050 \pm 0,000$ & 0,05 & 0,05 \\
\hline$A Q$ & $0,131 \pm 0,000$ & 0,131 & 0,03 \\
\hline$D Q$ & $0,030 \pm 0,000$ & 0,131 & 0,03 \\
\hline$A R$ & $0,581 \pm 0,123$ & 0,668 & 0.495 \\
\hline$D R$ & $0,025 \pm 0,007$ & 0,03 & 0,02 \\
\hline$A S$ & $0,484 \pm 0,054$ & 0,522 & 0,445 \\
\hline$D S$ & $0,020 \pm 0,000$ & 0,02 & 0,02 \\
\hline QRS & $0,068 \pm 0,004$ & 0,07 & 0,065 \\
\hline ST & $0,085 \pm 0,035$ & 0,11 & 0,06 \\
\hline$A T$ & $0,388 \pm 0,384$ & 0,659 & 0,117 \\
\hline$D T$ & $0,060 \pm 0,007$ & 0,065 & 0.055 \\
\hline
\end{tabular}

\begin{tabular}{|c|c|c|c|}
\hline \multicolumn{4}{|c|}{$20-29.9 \mathrm{Kg}$ (10 pacientes) } \\
\hline & Promedio & Máximo & Minimo \\
\hline$A P$ & $0,318 \pm 0,100$ & 0,438 & 0,163 \\
\hline$D P$ & $0,048 \pm 0,021$ & 0,095 & 0,03 \\
\hline$P R$ & $0,063 \pm 0,016$ & 0,095 & 0,045 \\
\hline$A Q$ & $0,535 \pm 0,405$ & 1,039 & 0,141 \\
\hline$D Q$ & $0,020 \pm 0,005$ & 0,025 & 0,015 \\
\hline$A R$ & $2,018 \pm 1,907$ & 5,261 & 0,159 \\
\hline$D R$ & $0,034 \pm 0,005$ & 0,045 & 0,03 \\
\hline AS & $0,494 \pm 0,278$ & 0,83 & 0,064 \\
\hline$D S$ & $0,023 \pm 0,015$ & 0,06 & 0,005 \\
\hline QRS & $0,066 \pm 0,014$ & 0,085 & 0,04 \\
\hline ST & $0,071 \pm 0,012$ & 0,085 & 0,055 \\
\hline AT & $0,580 \pm 0,563$ & 1,942 & 0,207 \\
\hline DT & $0,053 \pm 0,016$ & 0,07 & 0,03 \\
\hline \multicolumn{4}{|c|}{$>50 \mathrm{Kg}$ (1 paciente) } \\
\hline & Valor & Máximo & Mínimo \\
\hline$\overline{A P}$ & 0,354 & -- & --- \\
\hline$D P$ & 0,05 & - & - \\
\hline$P R$ & 0,03 & $-\cdots$ & $-\cdots$ \\
\hline$A Q$ & $\cdots$ & $-\cdots$ & $-\cdots$ \\
\hline$D Q$ & --- & -- & -- \\
\hline$A R$ & 0,91 & $\ldots$ & - \\
\hline$D R$ & 0,025 & -... & - \\
\hline AS & 0,217 & -..- & $-\cdots$ \\
\hline$D S$ & 0,015 & - & --.. \\
\hline$Q R S$ & 0,065 & --.- & -..- \\
\hline ST & 0,05 & - & - \\
\hline AT & 0,563 & - & --.- \\
\hline DT & 0,06 & --. & --.- \\
\hline
\end{tabular}

DERIVACIÓN DIII HEMBRAS

\begin{tabular}{c|c|c|c}
\hline \multicolumn{4}{|c}{$<10 \mathrm{Kg}$ (20 pacientes) } \\
\hline & Promedio & Máximo & Minimo \\
\hline \hline$A P$ & $0,262 \pm 0,080$ & 0,388 & 0,099 \\
$D P$ & $0,041 \pm 0,030$ & 0,15 & 0,02 \\
$P R$ & $0,046 \pm 0,014$ & 0,075 & 0,025 \\
$A Q$ & $0,119 \pm 0,082$ & 0,237 & 0,027 \\
$D Q$ & $0,012 \pm 0,004$ & 0,02 & 0,01 \\
$A R$ & $0,922 \pm 0,618$ & 2,491 & 0,224 \\
$D R$ & $0,025 \pm 0,005$ & 0,03 & 0,015 \\
$A S$ & $0,380 \pm 0,205$ & 0,857 & 0,049 \\
$D S$ & $0,021 \pm 0,005$ & 0,03 & 0,01 \\
QRS & $0,054 \pm 0,015$ & 0,085 & 0,025 \\
ST & $0,056 \pm 0,024$ & 0,095 & 0,02 \\
$A T$ & $0,368 \pm 0,240$ & 0,748 & 0,055 \\
$D T$ & $0,049 \pm 0,013$ & 0,06 & 0,02 \\
\hline
\end{tabular}

\begin{tabular}{c|c|c|c}
\hline \multicolumn{4}{|c}{$10-19.9 \mathrm{Kg}$ (9 pacientes) } \\
\hline & Promedio & Máximo & Minimo \\
\hline \hline AP & $0,275 \pm 0,107$ & 0,451 & 0,103 \\
DP & $0,033 \pm 0,022$ & 0,08 & 0,01 \\
$\boldsymbol{P R}$ & $0,054 \pm 0,020$ & 0,09 & 0,04 \\
AQ & $0,366 \pm 0,266$ & 0,673 & 0,207 \\
DQ & $0,013 \pm 0,004$ & 0,015 & 0,01 \\
AR & $0,961 \pm 0,698$ & 2,449 & 0,443 \\
DR & $0,031 \pm 0,007$ & 0,045 & 0,02 \\
AS & $0,312 \pm 0,117$ & 0,376 & 0,076 \\
DS & $0,032 \pm 0,023$ & 0,08 & 0,015 \\
QRS & $0,061 \pm 0,014$ & 0,085 & 0,045 \\
ST & $0,067 \pm 0,014$ & 0,09 & 0,05 \\
AT & $0,148 \pm 0,098$ & 0,349 & 0,036 \\
DT & $0,044 \pm 0,012$ & 0,055 & 0,03 \\
\hline
\end{tabular}

\begin{tabular}{c|c|c|c}
\hline \multicolumn{4}{|c}{$20-29.9 \mathrm{Kg}$ (15 pacientes) } \\
\hline & Promedio & Máximo & Minimo \\
\hline \hline$A P$ & $0,270 \pm 0,113$ & 0,423 & 0,055 \\
$D P$ & $0,037 \pm 0,014$ & 0,06 & 0,015 \\
$P R$ & $0,064 \pm 0,027$ & 0,115 & 0,035 \\
$A Q$ & $0,362 \pm 0,280$ & 0,683 & 0,167 \\
$D Q$ & $0,017 \pm 0,006$ & 0,02 & 0,01 \\
$A R$ & $0,939 \pm 0,663$ & 2,796 & 0,194 \\
$D R$ & $0,030 \pm 0,009$ & 0,05 & 0,015 \\
$A S$ & $0,284 \pm 0,241$ & 0,95 & 0,08 \\
$D S$ & $0,019 \pm 0,012$ & 0,04 & 0,005 \\
QRS & $0,078 \pm 0,086$ & 0,35 & 0,03 \\
ST & $0,075 \pm 0,024$ & 0,13 & 0,05 \\
AT & $0,317 \pm 0,240$ & 0,852 & 0,093 \\
$D T$ & $0,044 \pm 0,012$ & 0,065 & 0,03 \\
\hline
\end{tabular}

\begin{tabular}{c|c|c|c}
\hline \multicolumn{4}{|c}{$30-39,9 \mathrm{Kg}$ (6 pacientes) } \\
\hline & Promedio & Máximo & Mínimo \\
\hline \hline AP & $0,272 \pm 0,082$ & 0,414 & 0,213 \\
$D P$ & $0,041 \pm 0,012$ & 0,05 & 0,02 \\
$P R$ & $0,070 \pm 0,029$ & 0,105 & 0,04 \\
$A Q$ & $0,177 \pm 0,133$ & 0,355 & 0,021 \\
$D Q$ & $0,017 \pm 0,004$ & 0,02 & 0,01 \\
$A R$ & $1,143 \pm 0,529$ & 2,041 & 0,744 \\
$D R$ & $0,025 \pm 0,005$ & 0,03 & 0,02 \\
$A S$ & $0,346 \pm 0,202$ & 0,595 & 0,102 \\
$D S$ & $0,031 \pm 0,013$ & 0,05 & 0,025 \\
QRS & $0,061 \pm 0,012$ & 0,07 & 0,04 \\
ST & $0,080 \pm 0,014$ & 0,09 & 0,055 \\
AT & $0,402 \pm 0,148$ & 0,621 & 0,212 \\
$D T$ & $0,046 \pm 0,015$ & 0,055 & 0,02 \\
\hline
\end{tabular}

\begin{tabular}{|c|c|c|c|}
\hline \multicolumn{4}{|c|}{$>50 \mathrm{Kg}$ (2 pacientes) } \\
\hline & Promedio & Máximo & Mínimo \\
\hline$A P$ & $0,260 \pm 0,063$ & 0,304 & "0,216 \\
\hline$D P$ & $0,043 \pm 0,018$ & 0,055 & 0,03 \\
\hline$P R$ & $0,053 \pm 0,004$ & 0,055 & 0,05 \\
\hline$A Q$ & -..- & -.-- & $\ldots$ \\
\hline$D Q$ & $\ldots$ & $\ldots$ & $\ldots$ \\
\hline$A R$ & $1,708 \pm 0,590$ & 2,125 & 1,291 \\
\hline$D R$ & $0,045 \pm 0,000$ & 0,045 & 0,045 \\
\hline AS & $0,587 \pm 0,000$ & 0,587 & 0,587 \\
\hline DS & $0,025 \pm 0,000$ & 0,025 & 0,025 \\
\hline QRS & $0,058 \pm 0,018$ & 0,07 & 0,045 \\
\hline ST & $0,065 \pm 0,007$ & 0,07 & 0,06 \\
\hline AT & $0,409 \pm 0,052$ & 0,446 & 0,372 \\
\hline$D T$ & $0,048 \pm 0,004$ & 0,05 & 0,045 \\
\hline
\end{tabular}




\section{Anexo B}

\section{ECG MACHOS}

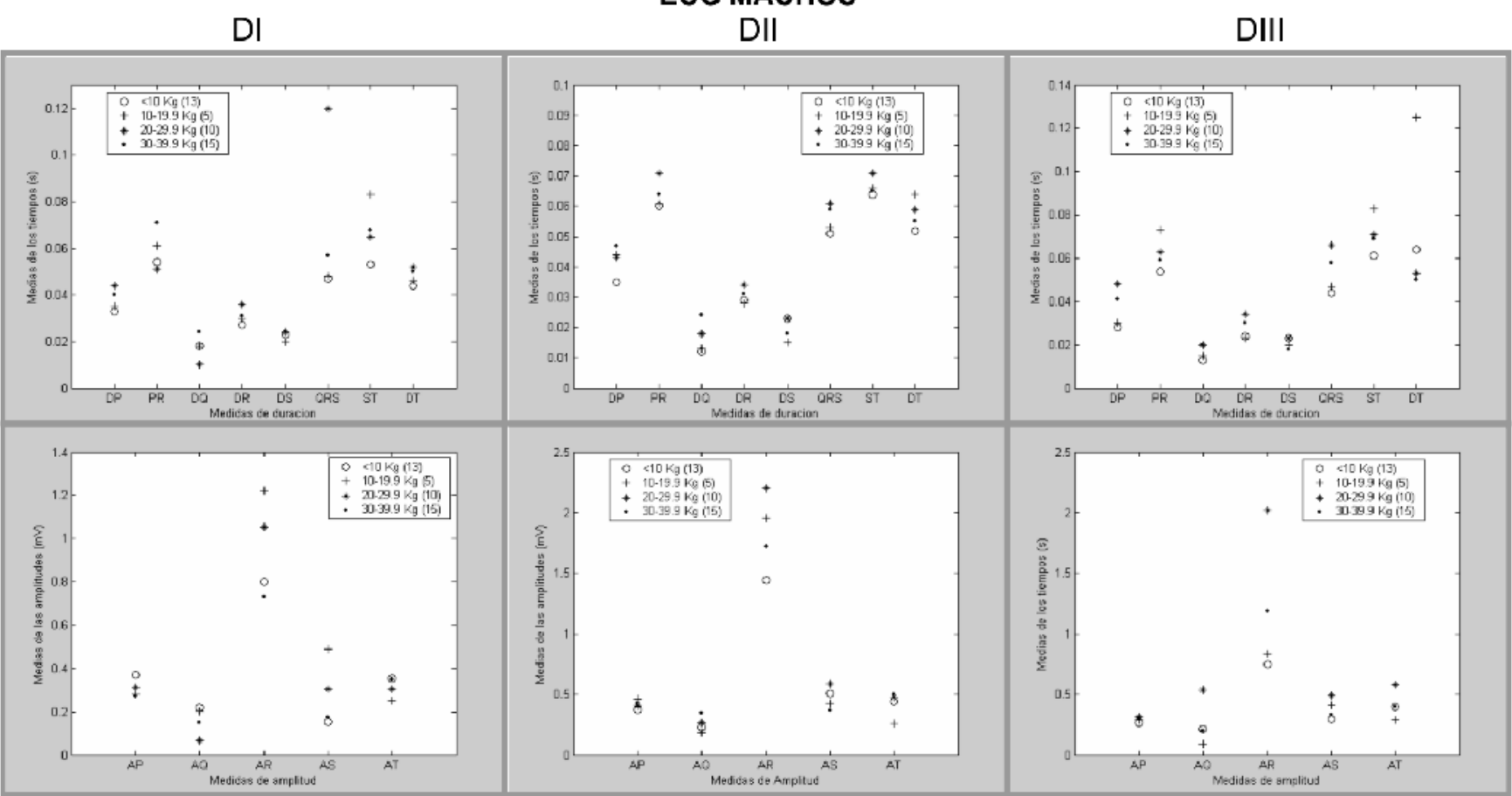

\section{ECG HEMBRAS}

DI

DII

DIII

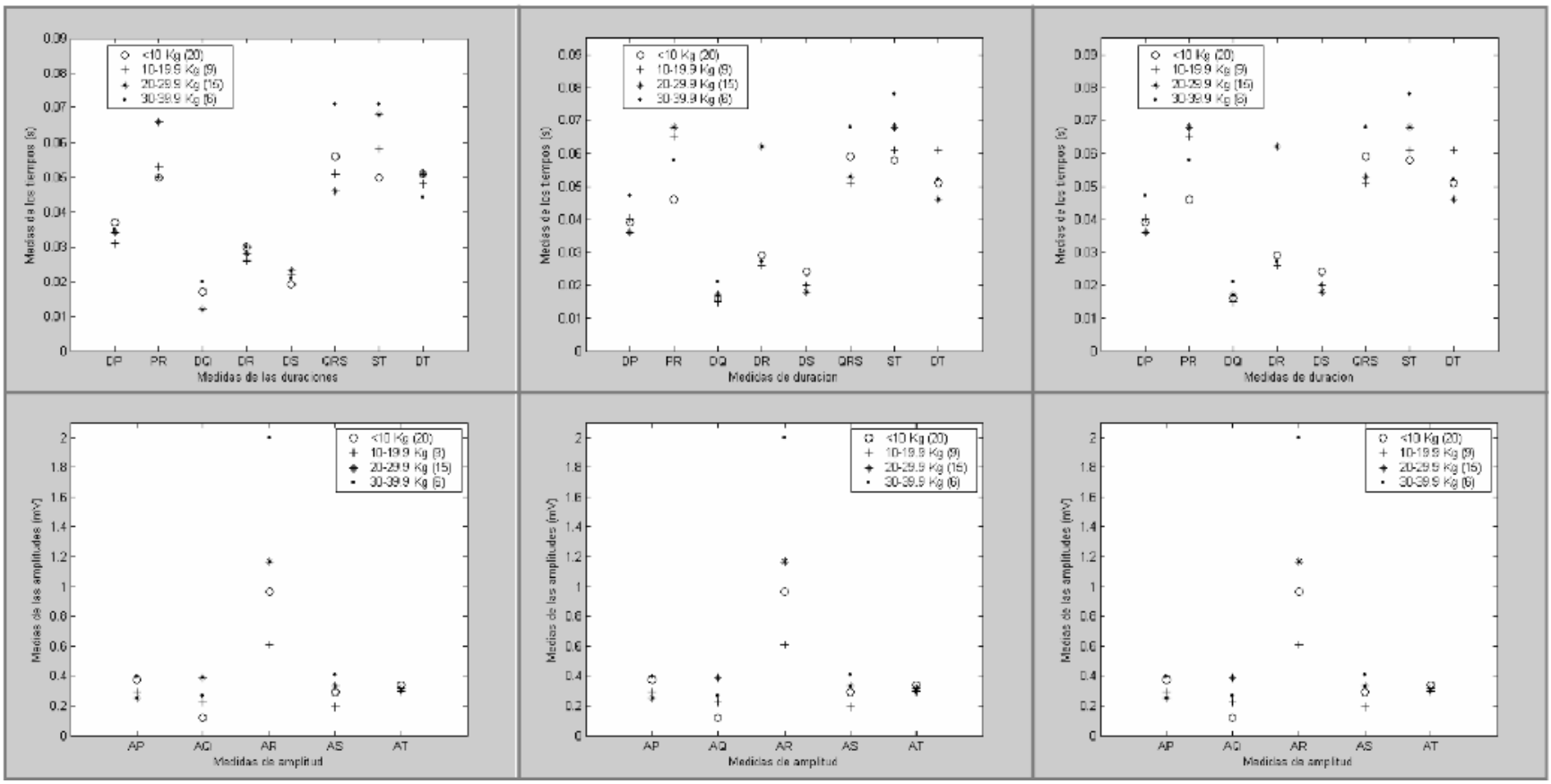

pacientes del mismo sexo pero con diferente peso, con base en las medidas de duración de las ondas (DR, PR, DQ, DR, DS, QRS, ST y DT), ya que las diferencias resultan ser muy pequeñas.
Las mayores diferencias entre pacientes del mismo sexo con diferente peso son más notorias en los resultados del examen de ecocardiografía, especialmente en lo que corresponde a los tamaños de la cá- 
mara en diástole (CamDiast), el volumen telediastólico (VolTeled) y el volumen de latido (VolLat).

\section{Discusión}

En los caninos muestreados, residentes en la ciudad de Bogotá, la amplitud y duración de la onda $\mathrm{P}$, que equivale a la actividad atrial derecha e izquierda, muestra valores superiores a la tabla de referencia, que reporta un máximo de $0.04 \mathrm{seg}$ de duración y $0,4 \mathrm{mV}$ de amplitud; la valoración ecocardiográfica demuestra que el aumento en amplitud y duración para la onda $\mathrm{P}$, no obedece a dilatación de las cámaras, cuyos valores se encuentran dentro de los datos reportados para caninos por peso, lo que no descarta de manera alguna, que las paredes atriales sean más gruesas o de mayor tamaño. En el protocolo utilizado para ecocardiografía canina, no se valora el tamaño de las paredes atriales, y solamente, se hace una comparación entre el diámetro de la aorta y del atrio izquierdo, para verificar si se presenta dilatación del atrio.

Con respecto al segmento $\mathrm{P}-\mathrm{Q}$, que es el tiempo necesario para que el impulso eléctrico pase del nodo atrioventricular (localizado en el atrio derecho) hasta el haz de his (localizado en los ventrículos), los valores obtenidos en la muestra se encuentran dentro de lo reportado en la tabla de referencia (0.06- $0.13 \mathrm{seg})$. El índice de Macraus utilizado en medicina humana, sugiere que la duración de la onda $\mathrm{P}$, debe ser semejante a la duración del segmento P-Q. Se observa este mismo comportamiento en los caninos objeto del estudio.

Para el complejo QRS, que se corresponde con la contracción ventricular derecha e izquierda, los datos obtenidos tienen valor agregado. Primero, en la tabla de referencia no se discriminan las diferentes ondas del trazado, como lo son, la onda $\mathrm{Q}$, la onda $\mathrm{R}$ y la onda $\mathrm{S}$, que son particularmente importantes para la derivación II y determinantes en el diagnóstico de patologías cardiacas; por otro lado, se obtienen datos para las tres derivaciones bipolares (la tabla de referencia sólo reporta la derivación II), y se definen las diferentes morfologías para cada una de las ondas y complejos, lo que se traduce en un interesante aporte al conocimiento del ECG canino.

Los datos obtenidos para la muestra, están dentro de lo reportado en amplitud para la onda $\mathrm{R}(3 \mathrm{mV}$ para razas grandes y $2 \mathrm{mV}$ para razas pequeñas), pero en el análisis ecocardiográfico, se verifica que el tamaño de la pared ventricular izquierda (en el protocolo ecocardiográfico se valoró el tamaño de la pared ventricular izquierda en sístole y diástole en modoM) es superior a la referencia para caninos, lo que demuestra paredes ventriculares izquierdas más gruesas para los caninos utilizados en la muestra y que son residentes en la ciudad de Bogotá. Esto tiene especial significancia clínica dado que, a pesar que la muestra posee paredes ventriculares izquierdas más grandes que la referencia para ercocardiografía, el promedio en amplitud para la onda R en el ECG, es mucho más bajo que el reportado, lo que lleva a pensar que, los valores reportados en la tabla de referencia, distan mucho de ser fiables en el diagnóstico de hipertrofias cardiacas.

Con respecto a la duración del complejo QRS, que se corresponde con el tiempo necesario total para la despolarización de los ventrículos, los datos obtenidos son mayores que la referencia $(0,06 \mathrm{seg}$ para razas grandes y $0,05 \mathrm{seg}$ para razas pequeñas) lo que se refuerza en el hallazgo de paredes ventriculares más grandes y mayor sensibilidad en el diagnóstico de hipertrofias, siempre y cuando, no se demuestren ritmos anormales que pueden modificar este valor, como por ejemplo, un fenómeno de aberrancia, un bloqueo de rama derecha y/o izquierda o un bloqueo atrioventricular.

La determinación del segmento S-T es de valor agregado para la base de datos, dado que la tabla de referencia no lo reporta. La duración de la onda $\mathrm{T}$ (repolarización ventricular), es semejante a la duración del segmento $\mathrm{S}-\mathrm{T}$, es decir que, el tiempo transcurrido desde la despolarización ventricular (terminación del complejo QRS) y el comienzo de la repolarización ventricular (comienzo de la onda $\mathrm{T}$ ), es semejante al tiempo requerido en la repolarización 
del ventrículo (duración onda T). De igual forma, los valores obtenidos para la duración de la onda $\mathrm{T}$, son de valor agregado dado que, por un lado, no existe tal determinación en la tabla de referencia, y por otra parte, el valor obtenido es similar al encontrado para la duración del complejo QRS (despolarización ventricular), lo que significa que el tiempo necesario para la despolarización ventricular, debe ser semejante al tiempo necesario para su repolarización.

En la muestra obtenida, se observa de manera clara lo reportado por la literatura en cuanto a que, la amplitud de la onda $\mathrm{T}$ no debe ser superior a un cuarto del tamaño de la onda R.

Este estudio demostró la importancia de hacer determinaciones locales y no limitarse a utilizar como guía diagnóstica lo reportado en trabajos foráneos, que son realizados en ambientes con características diferentes a las colombianas; por otra parte, la utilización de herramientas novedosas como la digitalización de la señal electrocardiográfica, le da mayor exactitud a las mediciones, lo que le confiere a la base de datos credibilidad para el clínico en ejercicio profesional.

\section{Referencias}

1. Belerenian G, Mucha C, Camacho J, Aparecido A. Afecciones Cardiovasculares en Pequeños Animales. Editorial Inter.Médica. Buenos Aires, Republica Argentina. 2001. 500p.

2. Kirk R. Terapéutica Veterinaria. Ed. Interamericana. 1994.

3. Ettinger, S. Tratado de Medicina Veterinaria. Editorial Inter.Médica. Buenos Aires - Argentina.1998. 431p.

4. Herding. Manual de Medicina Interna en Pequeñas Especies. Ed. Interamericana. 1996.

5. Fox, S. Textbook of Canine and Feline Cardiology. Saunders Co, Second Edition. 1999. 